\title{
LA TÉCNICA DE REJILLA COMO INSTRUMENTO DE EVALUACIÓN Y FORMULACIÓN DE HIPÓTESIS CLÍNICAS
}

\author{
GUILLEM FEIXAS, MARISOL DE LA FUENTE y JOAN MIQUEL SOLDEVILA
}

\author{
Universidad de Barcelona
}

(Aceptado en junio de 2003)

\begin{abstract}
El presente artículo se inicia con una presentación de la Técrica de Rejilla (TR), enmarcada en el contexto de la Teoría de los Constructos Personales (TCP). A continuación, se expone la aplicación de la TR al caso de Ana diagnosticada por trastorno depresivo mayor, bulimia nerviosa y trastorno de personalidad por evitación, además de presentar otros síntomas variados. Se eligio este caso por su complejidad clínica y por la manera en que se ven reflejados los síntomas de la paciente en su estructura cognitiva, puesta de manifiesto por la TR. A pesar de que Ana pidió ayuda por iniciativa propia, en las sesiones se muestra reticente a hablar de sí misma, por lo que la TR resultó ser un buen medio de aproximación a la visión del mundo de la cliente, y un instrumento útil para formular hipótesis clínicas.
\end{abstract}

Palabras clave: Técnica de la Rejilla, trastorno depresivo mayor, bulimia, trastorno de personalidad por evitación.

\section{Repertory Grid Technique as an assessment instrument for case formulation}

This paper begins with a presentation of the Repertory Grid Technique (RGT) within the framework of Personal Construct Theory. It follows with the description of the administration of a grid to Ana, a client diagnosed with major depressive disorder, bulimia nervosa, and avoidant personality disorder, as well as of other symptoms. This case is selected to illustrate the use of the RGT because of both its clinical complexity and the way in which her cognitive structure(as depicted by the RGT) is reflected in the presenting symptoms. Despite the fact that Ana eagerly requested help, during the therapy sessions she seems to be reluctant to talk about herself. The RGT proved to be a useful way of approaching her world view, and also a powerful instrument for case formulation.

Key words: Repertory Grid Technique, major depressive disorder, bulimia nervosa, avoidant personality disorder.

\section{INTRODUCCIÓN}

La Técnica de la Rejilla (TR) es un procedimiento de evaluación semi-estructurado (véase Feixas y Cornejo, 1996, Rivas y Marco, 1985) dirigido a evaluar los constructos personales del entrevistado, y generalmente se incluye dentro de las "técnicas subjetivas" (Feixas, 2003). Esto

Correspondencia: Guillem Feixas, Universidad de Barcelona, Departamento de Personalidad, Evaluación y Tratamiento Psicológicos, Passeig de Vall d'Hebron, 171, 08035 Barcelona.

Nota: Una versión preliminar de este trabajo fue presentada al VII Congreso Internacional de Constructivismo en Psicoterapia, Ginebra, 2000. significa que su misión es captar y comprender el modo en que una persona da sentido a su propia experiencia a través de sus propias palabras. Se trata, pues, de una técnica idiosincrásica, centrada en la persona o basada en una perspectiva «laica" (Hampson, 1982), que se diferencia de la "centrada en el investigador" porque parte de los constructos personales del entrevistado y no de los constructos teóricos del entrevistador.

La TR es una técnica de evaluación constructivista (Neimeyer, 1993). Se basa en el supuesto de que los seres humanos construimos unos significados con los que organizamos nuestro entorno físico y 
social. No podemos acceder, pues, a una realidad verdadera y única sino que múltiples construcciones de la realidad son posibles. La TR al igual que la técnica de la flecha descendente y el escalamiento (Botella y Feixas, 1998), se centra en explicitar el sistema de creencias de la persona y conocer cómo está organizado.

George Kelly (1955) creó la TR, que originalmente llamó "RepTest», en el contexto de su Teoría de los Constructos Personales (TCP). Esta teoría constituye actualmente un destacado precedente de la aproximación cognitivo-constructivista en psicoterapia (Feixas y Villegas, 2000; Neimeyer y Mahoney, 1995). Kelly, concibió al ser humano como un científico que elabora hipótesis sobre sí mismo, su entorno, los otros y en definitiva, sobre el mundo en el que vive; y que las pone a prueba constantemente en el ciclo de su experiencia (véase también Botella y Feixas, 1998; Kelly, 2001)

Las hipótesis personales están conformadas por dimensiones de significado o constructos, que consisten en la captación de una diferencia o un contraste en la experiencia del ser humano. El significado de una experiencia siempre implica un contraste, un opuesto. Por ejemplo, para apreciar que algo es suave (el significado de la experiencia de suavidad), hay que tener la experiencia de una textura áspera, y a la inversa. Para aprehender el significado de estas experiencias, necesitamos captar un contraste en la experiencia sensorial de la consistencia de los objetos que nos rodean. La captación de tal discrepancia, junto con las etiquetas que nos ofrece el lenguaje como "áspero" y "suave», nos permite organizar la experiencia de la aspereza de los objetos y dar sentido a una parte - ínfima en este caso- de nuestra vivencia del mundo.

Los constructos, como vemos, son abstractos y bipolares. Abstractos porque aunque surgen de la captación de las diferencias entre dos elementos luego se pueden aplicar a muchos otros; y bipolares porque surgen de un contraste entre dos elementos que se constituyen en sus dos polos opuestos. Pero además de ser bipolares, son dimensionales, puesto que existe una continuidad entre los polos.

Un constructo nos permite organizar la experiencia no sólo diferenciándola sino también integrándola, es decir, hallando similitudes. Así por ejemplo, uno de nuestros constructos personales puede ser "callado vs. hablador", que refleja la captación de una diferencia entre las personas; mas, al mismo tiempo, nos permite abstraer similitudes, por ejemplo: «mi hermana es habladora como mi madre, que es todo lo contrario a mi padre que es muy callado". Se han asimilado dos experiencias (la frecuencia del habla de madre y hermana) y se han distinguido de una tercera (la escasez del habla del padre).

Los constructos no son distinciones aisladas, se organizan en una estructura en red en la que se hallan interconectados unos con otros. Esta red es jerárquica: hay constructos que son más nucleares que otros $y$, por ello, ocupan posiciones supraordenadas. Los constructos nucleares hacen referencia a la identidad de la persona (¿quién soy yo en el mundo?) y, puesto que la identidad se construye en interacción con los otros, suelen configurar también el mapa de nuestro territorio interaccional. El núcleo del sistema tiene conexiones tan numerosas e intensas con el resto que los constructos nucleares son muy resistentes al cambio. Los seres humanos tendemos fuertemente a mantener nuestra capacidad predictiva y nuestra teoría implícita o red de significados porque sin ella estaríamos inmersos en un caos a merced de los acontecimientos; esto hace que en el intento de mantener la coherencia con nuestra estructura nuclear, prefiramos sufrir (si es necesario) antes que perder esa coherencia. 
La TR es un instrumento para explorar el sistema de constructos del entrevistado y su estructura. Formalmente, consiste en una tabla de doble entrada en la que los elementos o ejemplos significativos del área a explorar, se sitúan en las columnas y los constructos en las filas. En este artículo, se describe tanto el procedimiento de administración como su análisis psicológico, con especial atención en identificar aspectos que permitan comprender los significados implicados en los síntomas. Esta descripción se realiza en función del caso de Ana, hasta el punto de llegar a formular algunas hipótesis clínicas que permitan comprender los procesos psicológicos relacionados con su sintomatología y que puedan servir de inspiración para el diseño de la intervención psicológica.

\section{EL CASO DE ANA}

Ana es una mujer de 34 años, soltera y que trabaja como vigilante de seguridad. Acude a consulta privada derivada por el médico de familia con la orientación diagnóstica de trastorno depresivo mayor, bulimia nerviosa y trastorno de personalidad por evitación. Para entonces ya había recorrido un pequeño camino con profesionales de la salud mental y seguía tratamiento farmacológico con antidepresivos y ansiolíticos. Hacía dos meses había realizado su segunda tentativa de suicido tras la cual la ingresaron unas semanas.

En la primera sesión, nos explica que se siente muy triste, desilusionada y no tiene ganas de hacer nada. No duerme bien y está inquieta. Tiene sentimientos de rabia y resentimiento. Está harta del trabajo y se siente poco femenina. No ha tenido relaciones sentimentales significativas y le gustaría «tener novio». Su figura le preocupa ya que tiene sobrepeso, actualmente no realiza atracones pero no consigue sentirse bien con la comida. Tiene la sensación de no merecer nada bueno y cree que tiene lo que se merece. Hace tres años que no se encuentra bien, habla de una parte de sí misma que perdió entonces, una parte activa, que cree que ya no volverá jamás. Se muestra reservada y trasmite la sensación de que le cuesta dejarse ayudar, a pesar de que lo desea. Mantiene la distancia y parece desconfiada.

Ana vivía sola pero a consecuencia de su estado, vuelve al hogar paterno donde viven sus padres y sus dos hermanas pequeñas, María de 21 años y Sara de 20. Con sus padres no se entiende bien, especialmente con su padre, hacia quien siente vergüenza y resentimiento; con la madre se siente cohibida. Mantiene buena relación con su hermana pequeña pero no así con su hermano mayor, Javi de 35 años, que no vive en casa. Tiene pocos amigos y hace tiempo que no los ve pero piensa que estarán dispuestos a ayudarla cuando los necesite.

En la segunda sesión se pasa la TR, lo que resulta ser una buena estrategia para acercarnos al mundo de Ana, así como para desarrollar la alianza terapéutica. En la administración se establece una interacción entre paciente y terapeuta en torno a un objeto que es la rejilla, y se desarrolla, entre ambos, una dinámica de cooperación en una tarea conjunta, la elicitación de los constructos. Surgen significados personales y relacionales de la paciente de forma indirecta a través del «juego» de la rejilla, lo que resulta, para muchas personas, una forma más fácil y cómoda de hablar de sí mismos. Se consideró oportuno administrar la TR, además de por ser un instrumento de evaluación habitual para nosotros, por la dificultad de Ana para comunicarse con el terapeuta y establecer una relación de confianza. Nuestro objetivo era intentar conocerla de una manera que le provocara menos inquietud y que suavizara la 
barrera que parecía haberse establecido entre ella y nosotros.

\section{¿COMO APLICAMOS LA TÉCNICA DE REJILLA? LA REJILLA DE ANA}

En primer lugar, hay que recordar que la $T R$ es un instrumento muy flexible que admite muchas posibilidades diferentes de aplicación. Nosotros hemos seguido los planteamientos y orientaciones recogidos por Feixas y Cornejo (1996) a la hora de aplicar la TR a Ana.

El primer paso antes de administrar la $T R$, es tener claro su diseño. No se trata de un instrumento estandarizado sino que se adapta a los objetivos de su administración y al ámbito de su aplicación, sea este el clínico, el educativo, el empresarial o el de investigación. En nuestro ámbito, la psicología clínica y la psicoterapia, el diseño que nos resulta de mayor utilidad es la rejilla interpersonal y es el que desarrollamos en el presente texto.

La rejilla interpersonal se caracteriza por el tipo de elementos, una muestra representativa de las personas más significativas con las que se relaciona o se ha relacionado la paciente. Este tipo de selección se basa en el supuesto de que es en la construcción del si-mismo y de los otros donde se hallan los constructos más nucleares, aquellos que conforman y confieren identidad a la persona. $Y$ son estos constructos nucleares los que se asocian con los problemas psicológicos. Además de los elementos, hay otras tres cuestiones que implica el diseño interpersonal: 1) la selección de los constructos, que surgen del paciente y no vienen dados por el clínico, como en el caso de una típica investigación nomotética; 2) la selección del sistema de puntuación: se utiliza un sistema de intervalos, y el más adecuado para su posterior tratamiento matemático es una escala Likert de siete puntos en el que los extremos se corres- ponden a los polos; y por último, 3) la selección del número de rejillas a administrar en función de la perspectiva que adopte el clínico, bien longitudinal, bien transversal. Lo más habitual es administrar una rejilla como evaluación inicial pero resulta muy interesante utilizar varias rejillas para evaluar el cambio en el curso del proceso psicoterapéutico. En el caso de Ana, sólo se administró una rejilla al inicio de la terapia.

Una vez sabemos qué diseño de rejilla queremos administrar, pasamos a la fase de administración. La administración de la rejilla interpersonal se realiza mediante una entrevista estructurada entre clínico y paciente, en el marco de un clima relajado y cooperativo que facilite al paciente la expresión de sus constructos personales. La actitud del clínico es fundamental: debe mostrar su profundo respeto y deseo de comprender sin juzgar, lo cual también facilitará la alianza y el posterior proceso terapéutico.

En la administración pueden señalarse tres subfases. La primera es la selección de los elementos y viene determinada parcialmente por la anterior fase de diseño. Según el diseño interpersonal, los elementos serán las personas significativas de la vida del paciente y el propio paciente (elementos del sí-mismo): padres, hermanos, otros familiares que hayan sido relevantes, los mejores amigos de ambos sexos, parejas pasadas y actuales, otras figuras significativas como profesores, vecinos, etc., y también una persona nongrata, vivos o muertos, del pasado o del presente, etc. Quiénes forman parte de la rejilla lo decide el paciente en función de esta lista de roles que le plantea el entrevistador. Además, hay que incluir los elementos del sí mismo que tienen un especial interés psicoterapéutico. El elemento del sí-mismo imprescindible es el yo actual, al que se suma el yo-ideal («como me gustaría ser"). Hay otros elementos del sí mismo también de interés: por 
ejemplo, los temporales como "yo-antes», "yo-dentro-de-un-año»; los elementos relacionados con el síntoma como «yocon-ataques-de-pánico", "yo-cuando-consumo", etc.; y los elementos de roles como "yo-como-esposo", "yo-como-amiga», etc. El entrevistador sugiere alguno en particular según la problemática del paciente y lo que desee explorar.

En el caso de Ana, se seleccionan quince elementos que incluyen, además del "yo actual» y el "yo ideal», un elemento del sí mismo temporal ("yo antes»), su familia nuclear, tres buenas amigas, tres buenos amigos y un elemento ideal referido a una pareja sentimental ("pareja imaginaria»). Los elementos "yo antes" y "pareja imaginaria», responden a áreas de la paciente que el terapeuta considera importante explorar a partir de la información de la primera sesión. Recordemos que Ana hablaba de una parte perdida, de un antes y un después, y que también manifestaba cierta preocupación por no tener pareja actualmente.

Los elementos seleccionados, se anotan en la parte superior del protocolo de la rejilla (Figura 1), cada uno en una

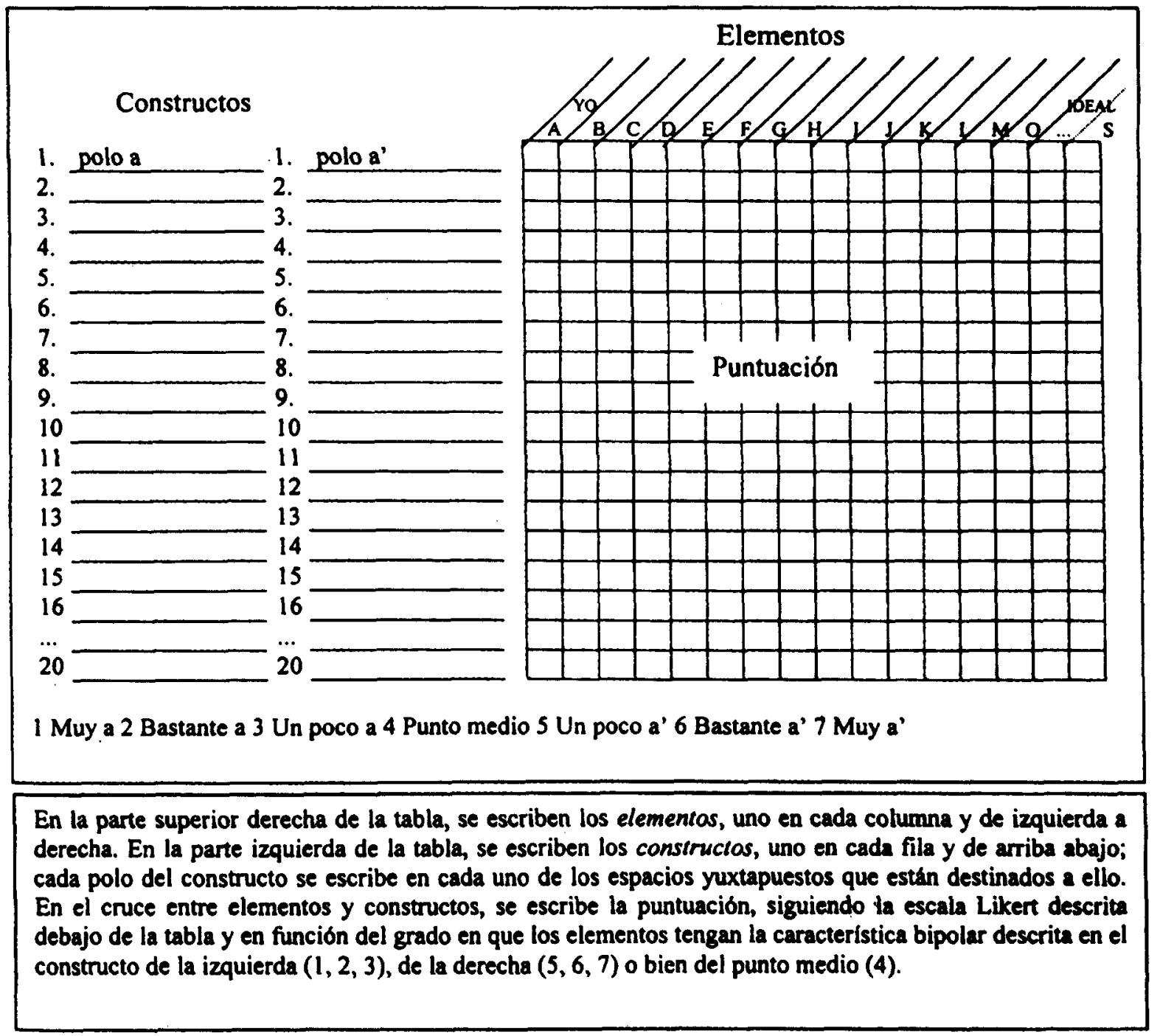

Figura 1. Protocolo de la Técnica de Rejilla adaptado de Feixas y Cornejo (1996) 
columna, (letras de la A a la S) reservando la primera columna para el "yo actual» y la última para el "yo ideal», por razones del tratamiento informático posterior. A su vez, los datos se apuntan en unas tarjetas en blanco que servirán para la subfase siguiente: la elicitación de los constructos. Cuando se agotan los elementos del paciente o cuando se llega a un número prefijado (por ejemplo, veinte que es lo aconsejable para nuestro posterior tratamiento informático) se pasa a la subfase siguiente.

Para la elicitación de constructos, se toman los pares de tarjetas que contienen los elementos. Ante cada díada, el clínico pregunta « ¿en qué se parecen el elemento ' $x$ ' $y$ el elemento ' $y$ ', en cuanto a su forma de ser, carácter o personalidad?» (por ejemplo, dada la díada padremadre: «¿en qué se parecen tu padre y tu madre, en su forma de ser.... ?»). El paciente responde la similitud que considere y ésta constituirá uno de los polos del constructo (por ejemplo, «mi padre y mi madre son generosos", "generoso" será uno de los polos del constructo) A continuación se pregunta: "¿y cuál es el contrario de esta característica?» (p.ej., «¿cuál sería el contrario de generoso? a lo que el paciente podría contestar: "tacaño") y de esta manera se obtiene el polo opuesto con el que se completa el constructo (en el ejemplo sería: "generoso vs. tacaño»). Los constructos elicitados se escriben en la entrada horizontal de la

\begin{tabular}{|c|c|c|c|}
\hline CONSTRUCTOS I 2 & $A B C D E F G H I J K L M N O$ & CONSTRUCTOS & ELEMENTOS \\
\hline 1-voluntari & 716161222131121 & 1-dejada & $A-Y O$ \\
\hline 2 -inteligen & 614121222121532 & 2 -tonta & $B-P A P A$ \\
\hline 3-tozuda & 7161111111431114 & 3-dejalleva & C-MAMA \\
\hline 4-realista & 216143432421156 & 4-fantasios & $D-H \quad 35$ \\
\hline 5-alegre & 767424224154433 & 5 -triste & $E-H \quad 20$ \\
\hline 6-intertrab & 7242722514511111 & 6 -desintrab & F-H 21 \\
\hline 7-alocada & 775514314444524 & 7 -nsabdisfr & G-AMIGA-1 \\
\hline 8 -espontane & 471717514441174 & 8 -responsab & $\mathrm{H}-\mathrm{AMIGA}-2$ \\
\hline 9-sincera & 244411111147741 & 9-hipocrita & $I-A M I G A-3$ \\
\hline 10-justa & 544566333447724 & 10-injusta & J-PAREJA-IM \\
\hline 11-equilibra & 717244353441741 & 11-inestable & K-AMIGO-1 \\
\hline 12-tranquila & 745373444411134 & 12-irada & L-AMIGO-2 \\
\hline 13-decisa & 717171444451114 & 13-indecisa & $M-A M I G O-3$ \\
\hline 14-idealista & 747414111465444 & 14-frustrada & $N-Y O-A N T E S$ \\
\hline 15-amantenat & 144433314144414 & 15-pasanat & O-YO-IDEAL \\
\hline 16-delqada & 747411111444542 & 16-qorda & \\
\hline
\end{tabular}

La primera columna corresponde a los polos de la izquierda (polos "a" según el protocolo de la figura 1) de cada constructo, mientras la tercera columna corresponde a los polos opuestos (polos "a"" en la figura 1). La cuarta columna corresponde a los elementos (columnas A... S según el protocolo de la fig. 1). Por último, la segunda columna es la puntuación resultante de describir el grado en que los elementos de las columnas (de $\mathrm{A}$ a $\mathrm{O}$ ) tienen las caracteristicas de los constructos de las filas (de 1 a 16), en función de la escala Likert de siete puntos (véase figuras 1,2 y 3 ).

Aclaración sobre las etiquetas de los constructos y elementos: 6- le interesa su trabajo vs. no le interesa su trabajo; 7- alocada vs no sabe disfrutar; 15- ama la naturaleza vs. pasa de la naturaleza; J- PAREJAIMAGINADA.

Figura 2. Matriz de los datos originales rejilla de ana, una vez introducidos al programa RECORD (Feixas y Cornejo, 1996) 
tabla, cada polo en uno de los espacios destinados a tal fin (véase Figura 1). Seguidamente se pregunta por más similitudes que pueda identificar, con las que se procede al igual que la primera, y a continuación, aun con el mismo par de elementos, se pregunta por la diferencia.

Ana no halló similitudes en la comparación de la primera díada presentada (padre y madre), por lo que se le preguntó seguidamente por las diferencias entre ellos ( $i$ en qué se diferencian tu padre y tu madre en cuanto a forma de ser, carácter o personalidad?») La respuesta aporta directamente los dos polos del constructo, "mi padre es voluntarioso y mi madre es dejada", dando lugar así al primer constructo: "voluntariosa vs. dejada». En la Figura 2 se pueden ver el conjunto de los constructos elicitados de Ana, un total de 16. En la primera columna de la matriz se hallan los polos de la izquierda, mientras que en la tercera columna se hallan sus opuestos.

La elicitación finaliza cuando se alcanza un número de constructos prefijado (alrededor de la veintena ya que un número mayor hace difícil de manejar el volumen de datos), o bien cuando el paciente comienza a repetir constructos ya utilizados y/o no puede elicitar más porque está agotado. Esto es lo que se conoce habitualmente como "punto de saturación».

La tercera y última subfase de la administración consiste en realizar la puntuacion. El paciente tiene que puntuar todos los elementos según una escala Likert de 1 a 7 . El extremo 1 de la escala hace referencia al polo escrito a la izquierda y el extremo 7 al polo escrito a la derecha; el punto medio (4) es una posición neutra que no se decanta por un polo u otro. Veamos, en la Figura 3, un ejemplo, tomando el primer constructo de la rejilla de Ana: "voluntariosa vs. dejada».

Al puntuar este primer constructo ("voluntariosa-dejada»), Ana refleja que ella ("yo actual») es muy dejada (puntuación 7), que su padre es muy voluntarioso (puntuación 1), que su madre es bastante dejada (puntuación 6) y que su ideal sería ser muy voluntariosa (puntuación 1). Gráficamente se puede representar como un vector (véase Figura 4). Los demás elementos (hermanos, amigos, pareja imaginaria y yo-antes) se van puntuando de la misma manera, en función de si tienden hacia un polo u otro y en qué medida.

Una vez tenemos la rejilla convenientemente cumplimentada (véase la rejilla de Ana en la Figura 2), pasamos al análisis de los datos. Una parte del análisis es cualitativo y se puede realizar directamente a partir de los datos originales, mientras que la otra parte es cuantitativa y necesita del tratamiento matemático mediante el programa RECORD v. $2.0^{2}$ (Feixas y Cornejo, 1996), especialmente ideado para el análisis de la TR. Este programa se caracteri-

2 En la actualidad RECORD se encuentra ya disponible la versión 4.0 en Internet: www.terapiacognitive.net/record

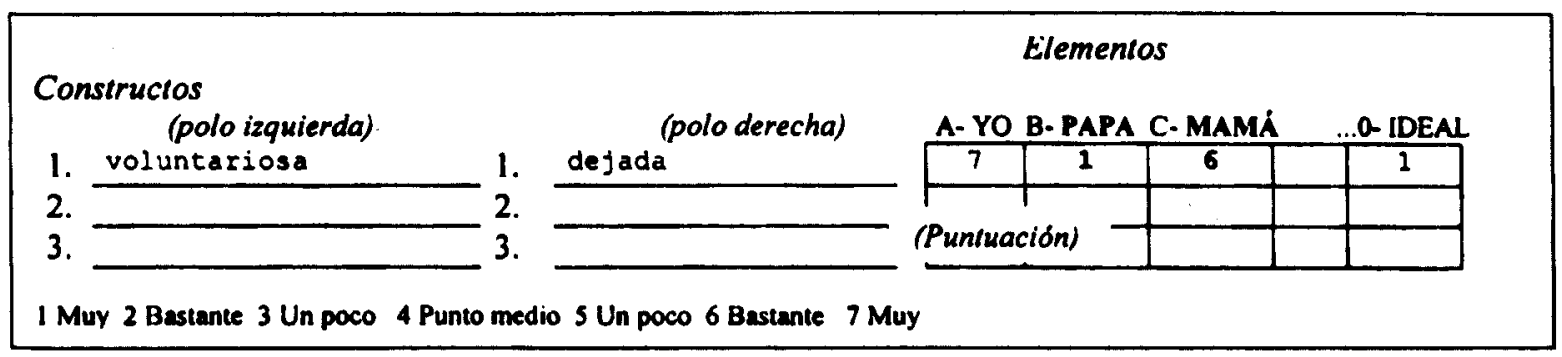

Figura 3. Detalle de la anotación de un constructo y su puntuación en la rejilla. 
CONSTRUCTO 1

Voluntariosa

1

2

3

4

PAPÁ/"YO IDEAL"

Figura 4. Representación vectorial de un constructo y su puntuación en la rejilla.

za por utilizar como técnica de factorialización, el Análisis de Correspondencias (AC), el cual usa como medida de similitud, la distancia «chi-2» y permite el análisis conjunto de constructos y elementos. Del análisis informático se obtienen una serie de listados que se interpretan para obtener datos e hipótesis relevantes para la clínica. En el siguiente apartado veremos la fase de interpretación, ejemplificado con la rejilla de Ana.

\section{¿QUÉ NOS DICE LA REJILLA DE ANA? LA INTERPRETACIÓN PSICOLÓGICA}

La tarea de interpretación es amplia y entretenida. Implica la revisión de múlti- ples aspectos psicológicos que nos aportarán información valiosa para el posterior proceso psicoterapéutico. Veamos cuáles son estos aspectos, empezando por los más directos y sencillos, y siguiendo por los más sofisticados.

\section{Autodefinición: ¿Cómo se define el} paciente con sus propias palabras?

A partir de los datos de la rejilla, podemos reconstruir la autodefinición del sujeto tomando los constructos en los que éste puntúa más, es decir, aquellos en los que el elemento "yo actual» obtiene valores extremos ( 1 y 2 ó 6 y 7). Para ello, el programa RECORD agrupa bajo el

Tabla 1. autodefinición y constructos discrepantes de Ana

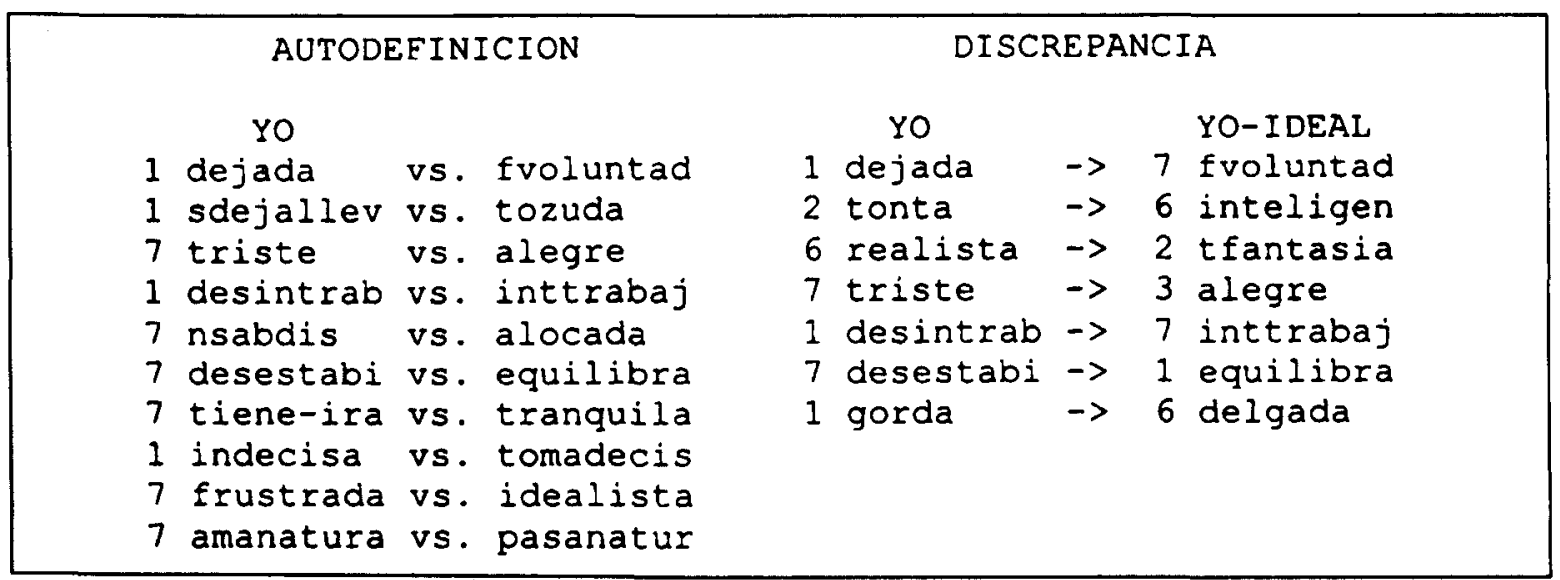

Bajo el título de "Autodefinición" se encuentran los constructos en los que uno de sus polos puntúa de forma extrema (1 o 7) para el "yo actual"; estos polos se situan a la izquierda, mientras que sus opuestos se situaan a la derecha. Bajo el titulo de "Discrepancia", se encuentran los constructos en los que el "yo actual" se sitúa en un polo y el "yo ideal" en el opuesto (entre ambos existe una diferencia minima de 4 puntos); los polos en los que se sitúa el "yo actual" se sitúan en la columna de la izquierda mientras los del "yo ideal" están a la derecha, todos ellos con su correspondiente puntuación. 
epígrafe "Autodefinición", todos los constructos en los que el "yo actual» puntúa 1 o 7 (véase Tabla 1). Las puntuaciones menos extremas de 2 y 6 que indica la tenencia de una característica en grado «bastante", las podemos identificar directamente en la matriz de datos originales (Figura 2) repasando la columna del «yo actual».

Una vez tenemos aislados los constructos más definitorios del "yo actual», podemos reconstruir narrativamente la autodefinición de nuestro paciente:

"Yo soy una persona muy dejada, que se deja llevar, triste, que no le interesa su trabajo, que no sabe disfrutar, desestabilizada, con mucha ira, indecisa, frustrada, que siente amor por la naturaleza y gorda. También me considero bastante tonta, realista y sincera».

Se obtiene una aproximación muy descriptiva y válida de la construcción del si-mismo del paciente, de su autoconcepto y de su identidad, lo que nos ayuda a verle como él se ve y a comprenderlo mejor en su sufrimiento. En el caso de Ana, vemos que la construcción de sí misma es muy negativa y llena de aspectos que desearía cambiar (constructos subrayados, a los que llamaremos discrepantes). Esto se comprende con el hecho de que, Ana no se siente ni feliz ni satisfecha de sí misma, lo que confiere sentido a sus síntomas depresivos. Asimismo, vemos que el constructo físico "gordadelgada", que se incluyó con el fin de explorar su preocupación por el peso, aparece en su autodefinición como un aspecto más de su identidad, algo que la define pero lo hace en el sentido no deseado (a ella le gustaría estar delgada), es un aspecto más del que no está satisfecha y guarda relación con los síntomas bulimicos.

Hay un único aspecto del que Ana se siente satisfecha y no le gustaría cambiar, es el ser «sincera» (en negrita), más adelante veremos que se trata de un constructo congruente. El resto de constructos de la autodefinición no señalados, son aspectos definitorios de Ana -en su mayoría de valor negativo- de los que tampoco se siente satisfecha, ya que su "yo ideal" no coincide con ellos (véase la columna del "yo ideal» en la Figura 2). A diferencia de los anteriores aspectos insatisfactorios, en éstos Ana no tiene claro cómo le gustaría ser (el ideal no se sitúa en el polo contrario sino que se queda en el punto medio); son los llamados constructos dilemáticos de los que hablaremos más adelante.

Recordemos que Ana hablaba de un antes y un después (operacionalizado en la rejilla con el elemento "yo antes»). Veamos, pues, cómo se veía con respecto a su visión actual:

"Yo era una persona con bastante fuerza de voluntad, muy tozuda, que le interesaba mucho su trabajo, bastante alocada, que tomaba decisiones e idealista, todo lo contrario a ahora. Además también era responsable y justa. Y, tanto antes como ahora, soy muy amante de la naturaleza».

Como vemos, el aspecto 'sincera' no formaba parte de su autodefinición. Por otro lado, vemos que el aspecto positivo 'amar la naturaleza' se mantiene en la Ana de antes y la Ana de ahora, constructo a tener en cuenta en el proceso terapéutico como un valor positivo que permanece.

\section{Comparación entre los elementos «yo actual» $y$ "yo ideal»}

En la Tabla 1 vemos la columna de «discrepancia», donde se señalan los constructos en los que el «yo actual» se sitúa en un polo y el "yo ideal» en el polo 
opuesto. Son los constructos discrepantes e indican aquellos aspectos que no le gustan a la persona de sí misma y que le gustaría cambiar en el sentido opuesto su ideal. Estos aspectos «insatisfactorios» o "puntos débiles» del sí mismo suelen coincidir con el motivo de consulta, y también muestran otras cuestiones a cambiar que antes no habían aparecido.

Los constructos discrepantes se pueden exponer de forma narrativa sugiriendo cómo los podría expresar el paciente. Veíamos cómo la autodefinición de Ana incluía todos los aspectos que no le gustan de sí misma; veamos ahora cómo le gustaría ser (polo ideal opuesto al polo actual):

"Al contrario de lo que soy (dejada, desinteresada por el trabajo, desestabilizada, tonta, realista, triste y gorda), me gustaría tener fuerza de voluntad, estar interesada por el trabajo, ser equilibrada, inteligente, fantasiosa, alegre, y delgada.»

Si comparamos esta hipotética narración de lo que le gustaría cambiar a Ana, con la construcción de sí misma, antes de estar deprimida ("yo antes»), vemos que sólo hay dos aspectos de cómo era antes que coinciden en cómo le gustaría ser: el tener 'fuerza de voluntad' y el tener 'interés por el trabajo'. Esto nos indica, por un lado, que estos dos aspectos son referencias del pasado a las que le gustaría volver $y$, por otro, que como era antes no es un modelo suficientemente adecuado de cómo le gustaría ser. Quizás antes tampoco estaba del todo contenta consigo misma; quizás los valores de antes no le sirvan ahora; o quizás ambas posibilidades... Lo que sí es cierto es que si miramos estos constructos en la matriz de datos (Figura 4), vemos que las puntuaciones del "yo ideal" para ellos, son puntuaciones de 4, es decir, su ideal no es ni un polo ni otro: no le gusta como es aho$\mathrm{ra}$, no quiere ser como era antes y no sabe cómo le gustaría ser. Son constructos poco funcionales (se tratarán más adelante como constructos dilemáticos).

Por otro lado, como ya habíamos comentado en la autodefinición, el único constructo en el que se siente satisfecha Ana es el del polo 'sincera' (opuesto a 'hipócrita'). Los constructos congruentes son aquellos en los que el «yo actual» y el "yo ideal» coinciden en el mismo polo (obtienen puntuaciones con una diferencia igual o menor que 1). Son los «puntos fuertes" de la persona, podrían ser nucleares para su identidad $y$, por tanto, muy resistentes al cambio. Esto hay que tenerlo en cuenta en el proceso terapéutico; son aspectos a validar y/o a proteger de posibles invalidaciones, puesto que constituyen aspectos fundamentales de la identidad de la persona. En el caso de Ana es muy indicativo que sólo haya surgido un constructo congruente, sólo un punto fuerte de sí misma, esta información nos ayuda a dar coherencia a sus graves síntomas depresivos.

\section{Dilemas implicativos y constructos dilemáticos: Dos modalidades de conflicto cognitivo}

Los dilemas implicativos son conflictos cognitivos en los que existe un aspecto del sí-mismo insatisfactorio que se desearía cambiar pero que, como se asocia fuertemente a aspectos positivos y satisfactorios de la identidad del paciente, es muy difícil de modificar (véase Feixas, Saúl, Ávila-Espada y Sánchez, 2001; también en Internet el Proyecto Multicéntrico Dilema: www.usal.es/tcp). El cambio es tan difícil porque implicaría el posible abandono de alguno de esos aspectos positivos y nucleares, lo cual representaría una amenaza para la identidad de la persona. Estas implicaciones dilemáticas 


\begin{tabular}{|lcc|}
\hline Constructo congruente: & $\begin{array}{c}\text { Sincera } \\
\text { ("yo actua"/"yo ideal") }\end{array}$ & $\begin{array}{c}\text { Hipócrita } \\
\text { (Polo indeseable) }\end{array}$ \\
$\begin{array}{l}\text { Constructo } \\
\text { discrepante }\end{array}$ & $\begin{array}{l}\text { Desinteresada } \\
\text { por el trabajo } \\
\text { ("yo actual") }\end{array}$ & $\begin{array}{c}\text { Intresada } \\
\text { por el trabajo } \\
\text { ("yo ideal") }\end{array}$ \\
\hline
\end{tabular}

Figura 5. Dilema implicativo de Ana

pueden extraerse cualitativamente de la matriz de correlaciones, pero la versión 4.0 del programa RECORD (véase nota 1) los detecta automáticamente. En Ana hallamos un dilema implicativo (véase Figura 5).

Según este dilema implicativo, a Ana le gustaría ser una persona 'interesada por su trabajo' - actualmente 'no tiene ningún interés' - pero eso implicaría ser 'hipócrita', algo absolutamente indeseable para ella porque ella es, ante todo, "sincera». Dicho de otra manera, para Ana las personan que se interesan por el trabajo son también hipócritas, por lo que realizar este cambio tiene el riesgo de dejar de ser sincera. Este conflicto cognitivo de Ana quizás podría explicar parte de su dificultad con el trabajo. Es necesario explorar qué significa ese tema del interés por el trabajo, en el contexto de sus valores representados en su sistema de constructos.

Por otro lado, están los constructos dilemáticos (de los que ya hemos ido hablando) que son también conflictos cognitivos. Estos constructos, a diferencia de los dilemas implicativos, no se basan en las implicaciones con otros constructos, sino que el dilema ocurre entre los dos polos de un único constructo.

Los constructos son dilemáticos cuando el "yo ideal" (la proyección de cómo le gustaría ser como persona) se sitúa en un punto medio (puntuación 4) sin deci- dirse por ninguno de los dos polos del constructo. Esos polos no constituyen alternativas deseables para el sujeto. En cierto modo, el constructo ha perdido su funcionalidad: la persona no sabe cómo le gustaría ser; no tiene referencias claras en su sistema cognitivo y necesitaría nuevas construcciones con las que orientarse. Los constructos dilemáticos son constructos que ya no le sirven para orientar sus esfuerzos, definir sus preferencias o tomar decisiones claras.

A veces ocurre que el "yo actual" también se sitúa en el punto neutro, junto al "yo ideal». En esos casos, la funcionalidad del constructo está aún más comprometida porque el constructo no sólo "no sirve" para saber cómo le gustaría ser sino que tampoco define cómo es ahora. La persona sólo puede decir de sí misma que no es ni un polo ni otro; es un constructo que no "funciona» para el sujeto, que necesitaría de nuevos constructos que le diesen mayor sentido.

Ana presenta numerosos constructos dilemáticos (véase Tabla 2). Su rejilla es atípica por la amplia presencia de este tipo de constructos, normalmente poco frecuentes. Este hallazgo otorga mayor coherencia y forma al profundo sufrimiento y desorientación que experimenta Ana y, en consecuencia, su diagnóstico de depresión mayor no resulta sorprendente.

Estos constructos dilemáticos constituyen en Ana el $50 \%$ de los constructos 
Tabla 2. Constructos dilemáticos de Ana

- Espontánea

- "yo actual» 4

- Tozuda

- «yo ideal» 4

- Alocada

- "yo ideal» 4

- Justa

- «yo ideal» 4

- Tranquila

- «yo ideal» 4

- Toma decisiones

- «yo ideal» 4

- Idealista

- «yo ideal» 4

- Ama la naturaleza

- «yo actual» 1 vs.

vs.

vs.

VS.

vs.

vs.

VS.

vs.
Responsable

- «yo ideal» 4

Se deja llevar "yo actual» 7

No sabe disfrutar

«yo actual» 7

Injusta

«yo actual» 5

Iracunda "yo actual» 7

Indecisa «yo actual» 7

Frustrada «yo actual" 7

Pasa de la naturaleza

"yo ideal» 4 elicitados, lo cual es de una magnitud muy llamativa. Sugiere que la red de significados de Ana, su sistema para moverse en el mundo y su identidad, están en crisis. En esta situación, la depresión puede constituir la respuesta coherente. Un objetivo terapéutico es conseguir que el sistema de Ana sea más funcional, es decir, le permita saber quien es y lo que quiere, además de saber ubicar a los demás.

\section{La construcción del sí mismo}

El estudio del sí mismo se basa en la comparación de las correlaciones entre los elementos que nos permiten estimar la relación que tiene el entrevistado consigo mismo y con su entorno social. Además, Feixas y Cornejo (1996) derivan de estas medidas unos perfiles tentativos sobre la construcción del sí mismo.

a) Autoestima: Correlación entre "yo actual» $y$ "yo ideal». Podemos utilizar la correlación entre las puntuaciones obtenidas para los elementos "yo actual» y "yo ideal», en los diferentes constructos, como un índice de autoestima que nos señala el grado de asociación entre estos elementos. Así, correlaciones positivas y altas indican una autoestima elevada ("yo actual" y "yo ideal" son muy similares, consiguen un alto grado de coincidencia, la persona es como quiere ser y, por tanto, está satisfecha consigo misma). Por el contrario, correlaciones muy bajas o negativas reflejan una baja autoestima. Esta forma de evaluar la autoestima parte de los constructos personales del paciente elicitados a través de la rejilla, de esta manera se respeta el punto de vista y la idiosincrasia del paciente. El hecho de no usar parámetros externos en la medida de la autoestima es relevante, dada la dificultad de evaluar y definir operacionalmente este constructo teórico.

Ana obtiene en este índice un valor negativo $(r=-0,57)$, lo que indica que su autoestima es bastante baja e incluso negativa: su forma de ser va en sentido contrario a cómo le gustaría ser. De la divergencia entre lo que es y lo que le gustaría ser, se deriva, en consecuencia, una gran insatisfacción consigo misma y 
un elevado malestar, lo que concuerda con el análisis cualitativo realizado en la autodefinición (recordemos que era una definición muy negativa y llena de aspectos insatisfactorio así como con la clínica).

b) Aislamiento social autopercibido: Correlación entre "yo actual» y "rotros». El elemento "otros» es un elemento construido a partir de la media de las puntuaciones otorgadas a todos los elementos, exceptuando el "yo actual" y el "yo ideal». La correlación entre estos elementos permite obtener la medida del grado de aislamiento social autopercibido, que se refiere a cómo se percibe de distinto el sujeto respecto a los demás.

Correlaciones negativas y elevadas, informan de un alto grado de aislamiento, es decir, indican que el paciente se ve muy diferente a las personas de su entorno, con quienes, probablemente, comparte pocos aspectos y experimenta cierto grado de aislamiento (sentimientos de soledad o incomprensión). Estas divergencias entre el «yo actual» y los «otros» se han observado con mayor frecuencia en personas deprimidas (Neimeyer y Feixas, 1992) y en mujeres víctimas de abusos sexuales (Harter, Neimeyer y Alexander, 1988). En ambos casos, este aislamiento ocurre en conjunción con una baja autoestima. Por otro lado, correlaciones altas y positivas son indicadoras de una buena identificación con las personas de su entorno, describirán sujetos con relaciones interpersonales fluidas, con amigos con quienes comparten numerosos aspectos.

En Ana, observamos un importante aislamiento social autopercibido $(r=-0,71)$. Se ve muy diferente a los demás, y esto le puede provocar un importante sentimiento de soledad y de sentirse un «bicho raro». Es una información más que aporta luz sobre otra faceta de su malestar. c) Adecuación percibida en los otros: Correlación entre "yo ideal» y «otros». La correlación de las puntuaciones de los elementos "yo ideal» y "otros» nos proporciona un índice de 'adecuación percibida en los otros', que indica el grado en que una persona ve a su familia y amigos (o personas significativas que han salido en la rejilla) convergentes con su "yo ideal». Correlaciones negativas o bajas, reflejan una fuerte insatisfacción con los otros, percibidos como «inadecuados». Hay que tener en cuenta que una percepción de gran "inadecuación» de los otros, podría ser también el resultado de un ideal excesivamente elevado y poco realista.

Ana obtiene una correlación positiva y moderada $(r=0,46)$, lo que nos indica que percibe a los otros como personas "adecuadas». Si comparamos este índice con el de autoestima, vemos que los otros se acercan más a su ideal que ella misma: «los otros son adecuados, yo no».

d) Perfiles del sí mismo. La interpretación conjunta de los diferentes índices comentados, nos conduce a los patrones o perfiles tentativos de la construcción del sí mismo (Feixas y Cornejo, 1996), orientándonos sobre el autoconcepto del paciente. Estos patrones son:

- Perfil de positividad: describe personas con una visión de ellos mismos y de los otros, positiva. Son personas que no presentarían conflictos ni áreas de su vida con déficits en su bienestar psicológico. Los índices de autoestima, de aislamiento social autopercibido y de adecuación percibida en los otros son todos altos y de signo positivo.

- Perfil de superioridad: describe sujetos que creen ser superiores a los otros. Los indices de aislamiento social autopercibido y de adecuación percibida en los otros son bajos 
o negativos, mientras que el de autoestima es positivo. Un perfil de este tipo puede señalar posibles trastornos de la personalidad narcisista o bien antisocial, también puede sugerir afecto hipomaníaco.

- Perfil de negatividad: describe sujetos pesimistas y podría ser indicativo de síntomas depresivos. El índice de aislamiento social autopercibido es positivo, mientras el de autoestima y el de adecuación percibida en los otros son negativos o de baja magnitud.

- Perfil de aislamiento: describe sujetos que presentan una tendencia a interpretar de manera negativa la información referente a ellos y de manera positiva la información referida a los otros. Los índices de autoestima y de aislamiento social autopercibido, resultan pobres 0 negativos, mientras que el de adecuación percibida en los otros resulta positivo.

- Perfil de resentimiento: describe sujetos que no tienen un buen autoconcepto de sí mismos y que, además, ven en los demás una falta de adecuación («los otros deberían ser de otra manera»). Es frecuente que este tipo de sujetos relacionen su malestar con las actitudes de los otros hacia ellos y experimenten sentimientos de rencor y rabia. Los índices de autoestima, aislamiento social autopercibido y de adecuación percibida en los otros son todos cercanos a cero o de signo negativo.

En una investigación sobre la resistencia (Feixas, Sánchez, Laso y Gómez-Jarabo, 2002) se estableció el punto de corte en $r=0,20$ para la identificación de estos perfiles, y esta variable resultó predictiva de los niveles de resistencia en algunos de sus valores. Por ejemplo, los pacientes con un perfil de superioridad presentaron más conductas de resistencia que el resto. Es decir, hay indicios que nos sugieren ciertas implicaciones clínicas en función del perfil identificado.

Interpretando los índices de Ana, observamos un patrón de aislamiento, según el cual Ana se ve de manera negativa y muy diferente a los demás, pudiendo tener la creencia de que ella es la única que está mal o de que es "rara»; asimismo sería probable que no compartiese aspectos relevantes con las personas significativas de su entorno $y$, en consecuencia, las relaciones interpersonales no fueran fluidas. El perfil de aislamiento es un perfil que suele corresponder a un sufrimiento de tipo depresivo, según sugieren Feixas y Cornejo, 1996; vemos que el caso de Ana concuerda con esta hipótesis.

\section{Análisis de la estructura cognitiva}

La diferenciación y la polarización son dos características fundamentales de la estructura cognitiva y nos aportan información sobre cómo es la estructura del paciente. La centralidad de los constructos nos informa sobre los constructos posiblemente nucleares del paciente, aproximándonos a una visión jerárquica de la estructura cognitiva o sistema de constructos personales.

\section{a) Diferenciación}

La diferenciación del sistema de constructos hace referencia a la complejidad cognitiva o al grado en que una persona puede construir sus experiencias desde diferentes puntos de vista. Cuanto más diferenciada es una estructura, más compleja es, y mayor capacidad tiene para poder integrar menos costosamente, los acontecimientos más diversos.

El índice de la diferenciación cogniti- 
va más reputado es el Porcentaje de la Varianza Explicada por el Primer Factor (PVEPF) resultante del análisis factorial. $\mathrm{Si}$ el porcentaje es elevado, es decir, si el primer factor explica mayoritariamente la varianza de los datos, indica que es una estructura simple ya que un solo factor es suficiente para dar buena cuenta de ella. $\mathrm{Si}$, por el contrario, el porcentaje es bajo, el factor explica poco, lo cual indica que es una estructura compleja y necesita de los subsiguientes factores para explicar suficientemente la varianza de los datos. Se estima, como orientación, que si el PVEPF es menor del $37 \%$, la estructura cognitiva es diferenciada, mientras que si es mayor de $47 \%$, la estructura es simple (Feixas, Bach, y Laso, 2003).

Ana obtiene un PVEPF de $38,36 \%$, según el cual su estructura cognitiva no es ni simple ni diferenciada de acuerdo con los datos tanto de población normal como clínica (Feixas, Bach, y Laso, 2003), en todo caso, tendería algo más hacia la diferenciación puesto que el primer factor explica relativamente poca varianza.

\section{b) Polarización}

La polarización hace referencia al grado en que una persona construye la realidad de forma dicotómica o polarizada. El índice se obtiene mediante el cálculo de la proporción total de puntuaciones extremas asignadas en la rejilla. La probabilidad teórica de obtener puntuaciones extremas, en una escala de siete puntos, es de $\mathbf{2 8 , 5 7 \%}$. En base a esta proporción, los porcentajes mayores tienden a describir estructuras rígidas y polarizadas, en las que hay blancos o negros, mientras que los porcentajes menores, describen estructuras en las que existe una mayor gama de grises. Neimeyer y Feixas (1992) encuentran que la polarización elevada se halla asociada a la severidad de los sínto- mas depresivos. En la clínica se observa que los depresivos suelen tener una visión polarizada de los acontecimientos y las personas.

Ana obtiene un índice de polarización elevado $(41,67 \%)$ con respecto al esperable por azar, lo que quiere decir que existen numerosas puntuaciones extremas en su rejilla. Esto nos indica que Ana tiene una estructura cognitiva rígida, en el sentido de extremista, y que construye el mundo, a sí misma y a los demás, de modo dicotómico del tipo "todo o nada» (Beck et al, 1979). Ello concuerda con la sintomatología depresiva que sufre.

\section{c) Centralidad de los constructos}

Este índice nos permite estimar la posición que los constructos pueden ocupar en la red de constructos personales del sujeto. El índice de intensidad de Bannister (1960), tomado constructo a constructo nos indica la centralidad de ese constructo. Se computa elevando al cuadrado la correlación de un constructo con cada uno de los demás, promediando estos resultados y extrayendo su raíz cuadrada. Normalmente, tal como sucede en este caso, los constructos con mayor intensidad son los que presentan mayor contribución absoluta (carga) a la determinación del eje o ejes más relevantes del análisis de correspondencias (CA). Cuanto mayor sea su peso o contribución, mayor se presume su centralidad.

En Ana, los constructos que hallamos con mayor intensidad y mayor contribución en el eje 1, son tres: «dejada vs. tener fuerza de voluntad», "dejarse llevar vs. tozuda", e "indecisa vs. toma decisiones». Por ello, podrían ser los más nucleares, lo cual implicaría que serían los constructos más resistentes y que conforman la identidad de Ana, sobre todo atendiendo a que se puntúa de forma extrema en estos constructos (véase Tabla 
1 o Figura 2). Sin embargo, no debemos olvidar que la rejilla es sólo una ventana al sistema de constructos del paciente y no es su sistema completo, estos indicadores de centralidad nos proporcionan únicamente una orientación sobre cuáles son los constructos más nucleares de los elicitados mediante la rejilla, no son determinantes absolutos.

Análisis específico de las implicaciones del constructo "gorda-delgada»

Dado los síntomas bulímicos de Ana, se creyó interesante explorar las relaciones del constructo "gorda-delgada», incluido para tal fin en la rejilla. En la Figura 6 se pueden apreciar de forma gráfica las relaciones del polo del constructo "gorda" (en base a la matriz de correlaciones), así como de otros fuertemente asociados con él y entre sí. Se observa una red de constructos con connotaciones negativas en el que todos se relacionan entre sí. El constructo gorda se asocia a los polos «tonta", "triste», "no sabe disfrutar", «frustrada" y «se deja llevar». Por lo tanto, para Ana el estar gorda implica mucho más que simplemente unos kilos de más; en consecuencia le dará gran importancia y lo intentará evitar en la medida de lo posible. En la medida en que se vayan articu- lando otros significados para el constructo 'gorda-delgada', Ana podría superar en gran medida su preocupación por la gordura o la delgadez.

\section{SÍNTESIS DE RESULTADOS, FORMULACIÓN DE HIPÓTESIS CLÍNICAS E IMPLICACIONES PARA LA TERAPIA}

Lo que más destaca en la rejilla de Ana es que la construcción de sí misma es muy negativa y llena de aspectos que desearía cambiar. No se siente bien consigo misma, lo cual se aprecia especialmente en el valor negativo del índice de autoestima. Su forma de ser va en sentido contrario a cómo le gustaría ser, por lo que debe experimentar una gran insatisfacción consigo misma y un elevado malestar. Esto da sentido a sus importantes síntomas depresivos. La autoestima y el autoconcepto serían áreas a trabajar en el proceso psicoterapéutico.

Además de un autoconcepto negativo y una baja estima de sí misma, hemos observado, a través de la rejilla, que Ana se ve muy diferente a los demás (aislamiento social autopercibido) y que percibe a los otros como personas «adecuadas» (adecuación percibida en los otros). Esto da lugar a un patrón de aislamiento,

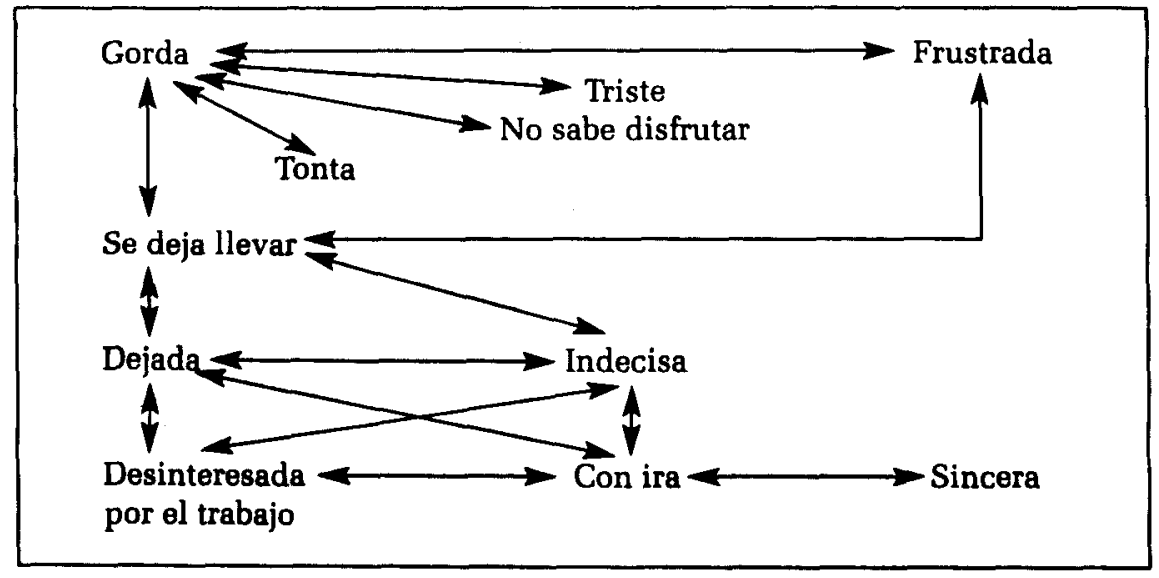

Figura 6. Red de constructos asociados a «gorda» 
en el que se pone de manifiesto que Ana se siente sola, distinta y peor que las personas de su entorno, con las que no comparte aspectos significativos. El perfil de aislamiento suele corresponder al tipo depresivo, lo que coincide perfectamente con la sintomatología de Ana. Está claro pues, que además de la autoestima es importante potenciar sus puntos de conexión con los demás, todo aquello que la haga sentir más cercana a ellos.

Otro índice que la literatura (p.ej., Neimeyer y Feixas, 1992) asocia a la sintomatología depresiva es el de polarización, que nos indica que Ana tiene una estructura cognitiva rígida y que se construye a sí misma, al mundo y a los demás, de un modo dicotómico y extremo. Flexibilizar su estructura cognitiva y conseguir unos constructos menos rígidos podrían constituirse en valiosos objetivos terapéuticos.

Algunos de los constructos más centrales de Ana son constructos que ella misma considera negativos, como "dejada», "dejarse llevar», e «indecisa», pero que forman parte de su identidad. A pesar de que le parezcan indeseables y desee cambiar, en el proceso terapéutico son constructos que hay que cuidar al ser parte de su identidad. Pueden resultar muy resistentes al cambio puesto que su invalidación supone la pérdida de una parte central de sí misma. Ana tiene una identidad negativa; pero no hay que pretender un cambio muy brusco, porque es la única que tiene. Para iniciar el cambio necesita validaciones; quizás más adelante pueda cambiar esos constructos indeseados, cuando tenga otros alternativos (Botella y Feixas, 1998). De momento, se aprecia una invalidación masiva del sistema de constructos de Ana, lo que confiere sentido a sus síntomas depresivos y ansiosos.

También hemos encontrado un dilema implicativa que nos permite formular una hipótesis acerca de las dificultades de
Ana por interesarse por el trabajo, puesto que esto lo asocia a ser «hipócrita».

Los constructos discrepantes conforman $43,75 \%$ de los constructos elicitados, mientras que los constructos congruentes representan sólo un 6,25\%. Esto viene a confirmar los resultados anteriores sobre su autoestima y autoconcepto. Pero lo más destacable, en el sentido de gravedad, es la gran cantidad de constructos dilemáticos (50\%).

La rejilla de Ana indica que su red de significados está en crisis. Su actual sistema de significados o de constructos no le sirve para realizar su función, anticipar los acontecimientos (Kelly, 1955) y darle un sentido de quién es y de cómo se ubica entre los demás. No le gusta como es; en algunos casos sabe cómo le gustaría ser (constructos discrepantes) y en otros -la mitad- no tiene una alternativa clara (constructos dilemáticos). Esto explicaría el profundo sufrimiento, fruto de la invalidación recurrente de sus construcciones, y la grave desorientación que experimenta Ana.

Tras ver todos estos datos, no sorprende la existencia de la sintomatología que sustenta la etiqueta de depresión mayor con la que es diagnosticada. También cobra mayor sentido el trastorno de personalidad por evitación, ya que ¿cómo no va evitar relacionarse con un mundo al que no puede predecir ni dar sentido, donde sus construcciones se invalidan constantemente? (Díaz, Feixas, Pellungrini, y Saúl, 2001). Es un mundo -y las personas que hay en él-que da miedo y, en consecuencia se evita. Kelly (1955; 2001) ya señaló la constricción como un recurso psicológico para reducir el campo perceptual (y las vivencias) a un ámbito reducido pero que al menos resulta predecible.

Un objetivo terapéutico fundamental sería conseguir que el sistema de constructos de Ana sea más complejo, más articulado, menos dicotómico y rígido, y 
que le permita predecir en gran medida los acontecimientos de su experiencia; en definitiva, más funcional. En el caso de Ana, un aspecto importante a trabajar para aumentar la capacidad predictiva de su sistema de constructos serían sus conflictos cognitivos, tanto los reflejados por cada uno de estos constructos dilemáticos, como el dilema implicativo que hemos mencionado anteriormente. En otro lugar (Feixas, Saúl, Ávila-Espada, A. y Sánchez, 2001; Feixas y Saúl, 2002; y en Internet: www.usal.es/tcp) hemos sugerido formas de intervención para abordar estos dilemas.

Ana sufre una invalidación global de su sistema de constructos personales. Esto provoca síntomas de depresión, pérdida de sentido de la vida, profundo sufrimiento, ideación suicida, comportamientos evitativos y una importante ansiedad que la conduce a atracones bulímicos. Hemos visto también como el constructo "gorda-delgada" conlleva importantes implicaciones de significado. En efecto, sería conveniente trabajar las asociaciones problemáticas ( frustrada», «triste», "no sabe disfrutar», «tonta», etc.) de este constructo en el proceso psicoterapéutico.

\section{A MODO DE CONCLUSIÓN}

A través de la TR han surgido algunas hipótesis de trabajo, áreas que creemos interesantes explorar así como posibles objetivos terapéuticos. Todo ello puede ser fundamental para diseñar la intervención y orientar el proceso terapéutico aunque no sean los únicos elementos a considerar. Ciertamente, el enfoque y diseño de la terapia escapa a los objetivos de este trabajo.

La rejilla es una técnica de evaluación que no aporta una simple valoración del paciente sino además importante información sobre su estructura cognitiva y sus relaciones personales, aparte de ser un elemento de interacción que favorece la alianza terapéutica y agiliza el proceso de evaluación. Finalmente, queremos destacar que la TR, además de ser un instrumento de evaluación y formulación de casos, ha mostrado su utilidad el campo de la investigación en psicoterapia (Winter, 2003). Por todo ello, consideramos que, aunque su uso es más extenso de lo que se manifiesta en la literatura de habla hispana (véase Luque, Rodríguez, y Camacho, $M, 1999)$, su potencial clínico y de investigación no esta siendo suficientemente aprovechado en nuestro campo.

\section{REFERENCIAS BIBLIOGRÁFICAS}

Bannister, D. (1960). Conceptual structure in thought-disordered schizophrenics. Journal of Mental Science, 106, 1230-1249.

Botella, L., y Feixas, G. (1998). Teoría de los constructos personales: Aplicaciones a la práctica psicológica. Barcelona: Laertes.

Beck, A., Rush, A., Shaw, B., y Emery, G. (1979). Terapia cognitiva de la depresión. Bilbao: Desclée de Brouwer, 1983.

Díaz, F., Feixas, G., Pellungrini, I., y Saúl, L.A. (2001). Cuando relacionarse amenaza la identidad: la fobia social desde un enfoque constructivista. Boletín de Psicología, 72, 43-55.

Feixas, G. (1999). Orientaciones para el análisis de la rejilla. Manuscrito no publicado, Universitat de Barcelona.

Feixas, G. (2003). Subjective methods. En R. Fernandez-Ballesteros (Ed.), The Enciclopedia of Psychological Assessment (Vol. II, pp. 937-943). London: Sage.

Feixas, G., Bach, L., y Laso, E. (2003). A comparative analysis of rep grid measures. Manuscrito sometido a publicación.

Feixas, G., y Cornejo, J.M. (1996). Manual de la técnica de rejilla mediante el programa RECORD ver. 2.0 ( $2^{a}$ ed.). Barcelona: Paidós.

Feixas, G., Procter, H., y Neimeyer, G. (1993). Líneas convergentes de evaluacion: contribuciones sistémicas y constructivistas. En 
G. Neimeyer (Comp.) (1993), Evaluación constructivista. Barcelona: Paidós, 1996.

Feixas, G., Sánchez, V., Laso, E., y GómezJarabo, G. (2002). La resistencia al cambio en psicoterapia: El papel de la reactancia, la construcción del sí mismo y el tipo de demanda. Análisis y Modificación de Conducta, 28, 235-286.

Feixas, G., y Saúl, L.A. (2002). Detection and analysis of cognitive conflicts: Implications for case formulation and therapy process. En T. Scrimali y L. Grimaldi (Eds.), Cognitive psychotherapy toward a new millennium (pp. 173-176). New York: Kluwer/Plenum.

Feixas, G., Saúl, L.A., Ávila-Espada, A., y Sánchez, V. (2001). Implicaciones terapéuticas de los conflictos cognitivos. Revista Argentina de Clínica Psicológica, X, 5-13.

Feixas, G., y Villegas, M. (2000). Constructivismo y Psicoterapia ( $3^{\mathrm{a}} \mathrm{ed}$. rev.). Bilbao: Desclée de Brouwer.

Hampson, S. (1982). La construcción de la personalidad. Barcelona: Paidós, 1986.

Harter, S., Alexander, P.C., y Neimeyer, R.A. (1988). Long term effects of incestuous child abuse in college women: Social adjustment, social cognition, and family characteristics. Journal of Consulting and Clinical Psychology, 56, 5-8.

Kelly, G.A. (1955/1991). The psychology of personal constructs (2 vols.). London: Routledge.

Kelly, G.A. (2001). Psicología de los constructos personales: Textos escogidos (B. Maher, compilación en inglés; G. Feixas, editor). Barcelona: Paidós.

Luque, A., Rodríguez, J., y Camacho, M. (1999). El uso de la rejilla: revisión. Anales de Psiquiatría, 15, 246-252.

Neimeyer, G. (Comp.) (1993). Evaluación constructivista. Barcelona: Paidós, 1996.

Neimeyer, R.A., y Feixas, G. (1992). Cognitive assessment in depression: A comparison of some existing measures. European Journal of Psychological Assessment, 8, 4756.

Neimeyer, R., y Mahoney. M. (Comps.) (1995). Constructivismo en psicoterapia. Barcelona: Paidós, 1998.

Rivas, F., y Marco, R. (1985). Evaluación conductual subjetiva: La técnica de rejilla. Valencia: Centro Editorial de Servicios y Publicaciones Universitarias.

Winter, D. (1993). Repertory grid technique as a psychotherapy research measure. Psychotherapy Research, 13, 25-42. 